\title{
Analysis of Raman Spectra by Using Deep Learning Methods in the Identification of Marine Pathogens
}

\author{
Shixiang Yu, Xin Li, Weilai Lu, Hanfei Li, Yu Vincent Fu,* and Fanghua Liu*
}

Cite This: Anal. Chem. 2021, 93, 11089-11098

Read Online

ACCESS | Llll Metrics \& More

ABSTRACT: The need for efficient and accurate identification of pathogens in seafood and the environment has become increasingly urgent, given the current global pandemic. Traditional methods are not only time consuming but also lead to sample wastage. Here, we have proposed two new methods that involve Raman spectroscopy combined with a long short-term memory (LSTM) neural network and compared them with a method using a normal convolutional neural network $(\mathrm{CNN})$. We used eight strains isolated from the marine organism Urechis unicinctus, including four kinds of pathogens. After the models were configured and trained, the LSTM methods that we proposed achieved average isolation-level accuracies exceeding 94\%, not only meeting the requirement for identification but also indicating that the proposed methods were faster and more accurate than the normal CNN models. Finally, through a computational approach, we designed a loss function to explore the mechanism reflected by the Raman data, finding the Raman segments that most likely exhibited the characteristics of nucleic acids. These novel experimental results provide insights for developing additional deep learning methods to accurately analyze complex Raman data.

\section{INTRODUCTION}

Urechis unicinctus, commonly known as the sea intestine, attaches to Echiuroidea ${ }^{1}$ and is a bentonic organism that commonly inhabits the intertidal zone and sublittoral zone along the coasts of the Huanghai Sea and the Bohai Sea. It possesses high culinary and medical value. ${ }^{2}$ In recent years, pollution of the marine environment due to the usage of chemicals in cultivation, especially the abuse of antibiotics, has resulted in the disappearance and extinction of a large number of organisms, and the ecological environment has been severely damaged, which is not conducive to the sustainable development of $U$. unicinctus aquaculture. Studies on intestinal bacteria, especially pathogenic bacteria, are becoming increasingly important and urgent. ${ }^{3}$ However, to date, no study on the diseases of $U$. unicinctus larvae has been conducted. ${ }^{4}$ In this study, eight kinds of bacteria, including four pathogens, were isolated by the plate streaking method from the intestine of diseased $U$. unicinctus larvae to provide an etiological theoretical basis for the study of the diseases of $U$. unicinctus larvae. However, the current diagnostic methods used to detect and identify bacteria are time consuming since at least several days are required to culture bacteria. Therefore, new rapid and culture-free methods need to be established for the detection and identification of bacteria. ${ }^{5-12}$

In recent years, biosensors have been widely used in medicine-related fields for rapid diagnosis. Since bacteria show unique biological characteristics in Raman spectra, Raman spectroscopy has the potential to identify species, and single cells can be analyzed. ${ }^{13-21}$ Subtle differences in the corresponding Raman spectra can also occur due to biochemical differences among different bacteria. At present, the complex neural network models represented by deep learning have become an important topic in artificial intelligence research. ${ }^{22}$ Their powerful characterization ability enables computers with complete learning and judgment similar to the human brain and, in some aspects, even imparts a decision-making ability beyond that of the human brain. Convolutional neural networks (CNNs) are typical feedforward neural networks and constitute an important branch of deep learning algorithms. By virtue of two important features, that is, local connection and value sharing, the learning and classification ability of the CNN model is far superior to that of traditional neural networks in terms of applicability, offering significant advantages in many fields. ${ }^{23-34}$ The CNN model

Received: January 29, 2021

Accepted: July 26, 2021

Published: August 2, 2021 
has been used by researchers to classify Raman spectra and has achieved good results, but the common application of CNNs combined with Raman spectroscopy still has many limitations. For example, while the number of strains to be classified is constantly increasing, the accuracy of classification is dramatically decreasing. The current solution is to either use a better algorithm or increase the depth of the network, but both of these solutions increase the model training time. At the same time, deep learning models are called "black box" models, and the chemical characteristics responsible for classification cannot be known. Therefore, we attempted to address the low classification accuracy, long training time, and unknown mechanism behind the models by designing more advanced algorithms. Recurrent neural networks (RNNs) can memorize the previous sequence input and apply it to a later calculation, but they cannot solve the long-term dependence problem. The long short-term memory (LSTM) model can avoid the problems of gradient disappearance through a gating unit system of model parameters and control information flow, making the prediction of time series information more accurate. $^{35}$

Many studies are currently attempting to explore the mapping of Raman data at the strain level due to the Raman characteristic peaks presumably belonging to a mixed feature, which leads to this mapping being difficult, particularly the analysis via analytical chemistry. We, therefore, designed our own loss function to explore the mechanism reflected by the Raman data by combining computational approaches. Raman segments that most likely belonged to the characteristic nucleic acids were subsequently found.

\section{METHODS}

Dissection of Diseased $U$. unicinctus Larvae and Isolation of Intestinal Pathogens. Diseased U. unicinctus larvae were collected from the Yantai Eastern Marine Yunxi Aquaculture Base in Shandong Province at a rate of 3000$4000 / \mathrm{kg}$. The diseased U. unicinctus larvae showed slow movement, floating from their cave under the sand to the surface of the sand, and had dark-red skin. After flushing with sterile water 3 times, the diseased $U$. unicinctus larvae were placed in a pallet on a clean bench that had been disinfected at high temperature for dissection under aseptic conditions. After separation, an intestine of proper length was put in a $1.5 \mathrm{~mL}$ centrifuge tube to which sterile normal saline was added and then blended. Then, $50 \mu \mathrm{L}$ of the mixed liquor on average was applied to solid Luria broth (LB) culture medium, which was placed in a $30{ }^{\circ} \mathrm{C}$ greenhouse to be cultured for $36 \mathrm{~h}$. The dominant single colony was then inoculated in $5 \mathrm{~mL}$ of liquid LB medium and cultured at a constant temperature of $30^{\circ} \mathrm{C}$ for $24 \mathrm{~h}$. After three rounds of separation and purification by repeated streaking, single colonies were selected from the liquid medium to be prepared as a fresh bacterial liquid. Glycerin at a volume fraction of $30 \%$ was added to the fresh bacterial liquid (logarithmic phase), which was then placed in a refrigerator at $-80^{\circ} \mathrm{C}$ for future use and appraisal. The above process was repeated to obtain 8 kinds of pure bacteria. ${ }^{36-38}$

16S rRNA Gene Sequence Analysis and Construction of a Phylogenetic Tree of the Predominant Bacteria. A single colony was selected with sterile tips, placed in $100 \mu \mathrm{L}$ of sterile water (in a polymerase chain reaction (PCR) tube), and incubated in a $100{ }^{\circ} \mathrm{C}$ water bath for $10 \mathrm{~min}$. Then, the tube was centrifuged for $3 \mathrm{~min}$ at a rotational speed of $7200 \mathrm{rpm}$. The supernatant was taken as the PCR template. PCR amplification was performed with universal primers for the bacterial 16S rRNA gene (synthesized by Shanghai Yingwei Jieji Biotechnology Company). The forward primer was $27 \mathrm{~F}$ ( $5^{\prime}$-AGAGTTTGATCCTGGCTCAG-3'), the reverse primer was 1492R (5'-TACGGCTACCTTGTTACGACTT-3'), and the PCR system $(50 \mu \mathrm{L})$ was as follows: $10 \times$ Ex Taq buffer 5 $\mu \mathrm{L}$, dNTPs $4 \mu \mathrm{L}$, 27F $2 \mu \mathrm{L}, 1492 \mathrm{R} 2 \mu \mathrm{L}$, ExTaq $0.25 \mu \mathrm{L}$, and sterile water $34.75 \mu \mathrm{L}$. The PCR conditions were as follows: pre-denaturation at $95{ }^{\circ} \mathrm{C}$ for $5 \mathrm{~min} ; 30$ cycles of $94{ }^{\circ} \mathrm{C}$ for 1 min, $57^{\circ} \mathrm{C}$ for $1 \mathrm{~min}$, and $72{ }^{\circ} \mathrm{C}$ for $2 \mathrm{~min}$; and $72{ }^{\circ} \mathrm{C}$ for 10 min. After detection with agarose gel electrophoresis, the PCR products were sent to Shanghai Yingwei Jieji Biotechnology Company for single-read sequencing. If a product was detected to represent a pure strain, the other end was also tested. Finally, the results were spliced to obtain a sequence on which BLAST analysis was conducted with the nucleotide sequences in GenBank to determine the sequence with the highest homology. ClustalX1.83 was adopted to perform multiplesequence analysis, and MEGA $\mathrm{X}$ software was adopted to construct the phylogenetic tree with the neighbor-joining method. Then, the literature was consulted to determine the pathogenicity of the strain, and the potential pathogens were tested for reinfection to determine the pathogenicity. ${ }^{39-41}$

Manual Infection Resumption Test. Twenty healthy $U$. unicinctus larvae were selected and placed in six $1 \mathrm{~L}$ flasks for 3 days, and three of the flasks were selected at random as the treatment group, while the other three were selected as the control group with the same controlled growth conditions (temperature: $23^{\circ} \mathrm{C}$, salt content: $25, \mathrm{pH}: 6.3$, and continuous oxygenation). If no diseased $U$. unicinctus larvae were observed within 3 days, appropriate amounts of fungal suspensions were injected into the treatment group, and the pathogenesis and death conditions of the treatment group and control group were observed. ${ }^{42}$ The same tests were performed on each potentially pathogenic strain.

Raman Sample Preparation. Single colonies of each pure culture from the LB agar plate were inoculated in $10 \mathrm{~mL}$ of $\mathrm{LB}$ medium, followed by cultivation overnight at $37{ }^{\circ} \mathrm{C}$ in a shaking incubator. ${ }^{43}$ After $12 \mathrm{~h}$ of incubation, the cells were harvested by centrifugation at $12,000 \mathrm{rpm}$ for $2 \mathrm{~min}$ and resuspended in sterile physiological saline twice. Each strain was cultured independently in at least three different batches for biological replicates. Raman data for each bacterium were collected in three independent sets of experiments for model training or model evaluation.

Raman Spectroscopy. The excitation wavelength selected for the study was $785 \mathrm{~nm}$ (originating from a diode laser), and a $100 \times$ microscope objective was used to focus the excitation light onto the sample. In this method, scattered Raman signals pass through a monochromator, where they are diffracted using a 600 -groove $/ \mathrm{mm}$ grating and collected with a thermoelectrically cooled charge-coupled device (CCD) camera with a spectrum resolution of $6 \mathrm{~cm}^{-1}$. Wavenumber calibration was performed using microsphere standard peaks at 620.9, 1001.4, and $1602.3 \mathrm{~cm}^{-1}$ before acquiring the sample spectra. The Raman spectra, including the spectra of the background and pathogens, were acquired for $60 \mathrm{~s}$ at different positions. We acquired 20 spectra of the background and $\sim 150$ spectra of the pathogens. Appropriate preprocessing methods can effectively improve the applicability of the model. Then, data containing cosmic rays were deleted to obtain the results shown in the figure. We used the subtraction method to remove the background data. Then, a Savitzky-Golay filter was used for 
a.
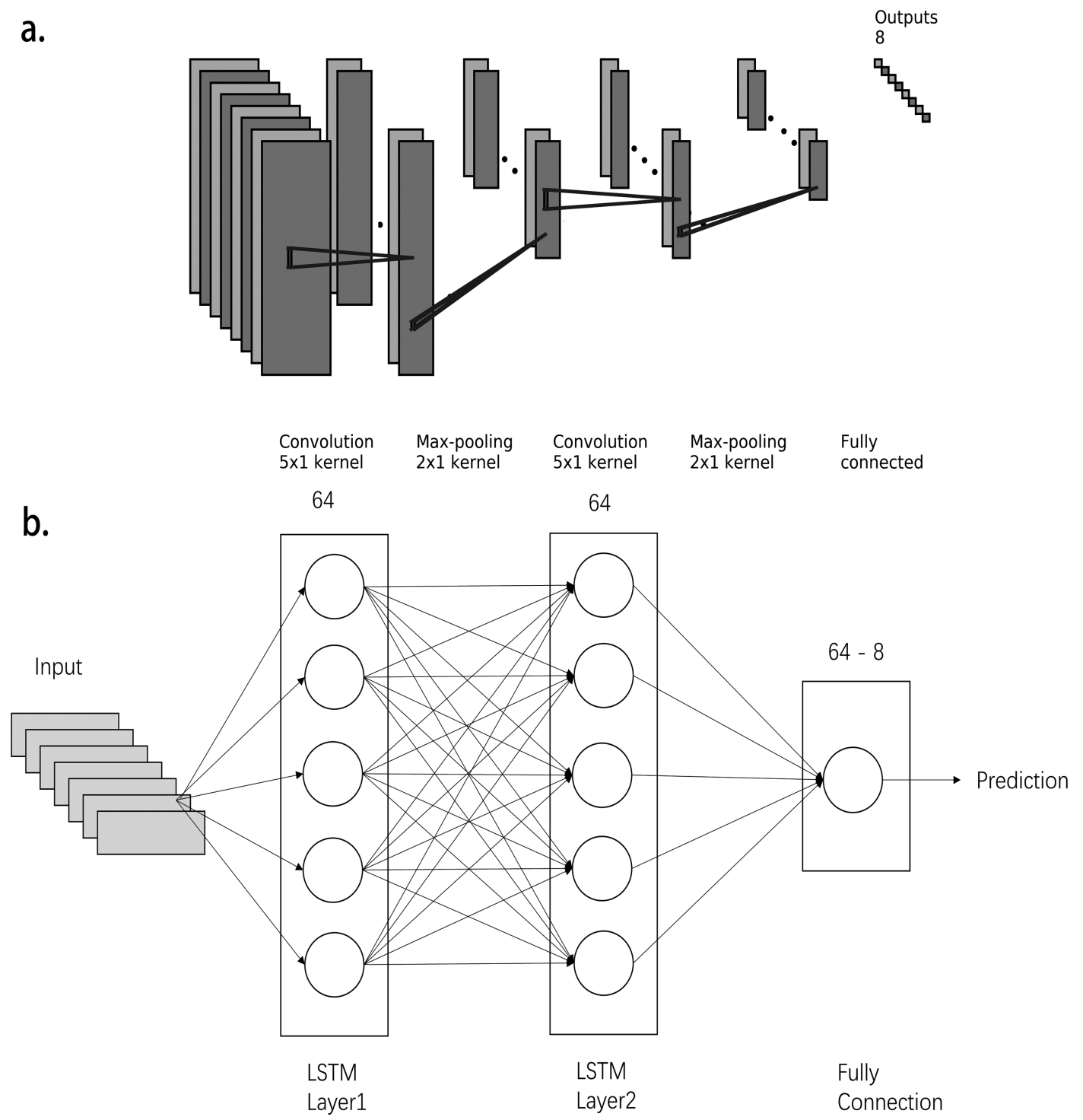

Figure 1. Deep learning model architecture. (a) First, we designed a CNN that consists of three layers. The first two layers are used to extract the features of the Raman data, and each layer is a combination of a convolution layer and a pooling layer. The last layer is a fully connected neural network layer for classification. The convolution layers contain 16 and 32 neurons, and the convolution filter of each layer is $1 \times 5$. Then, after being activated by the ReLU function, the network enters the maximum pooling layer and pools by $1 \times 2$. Finally, the eight-dimensional data are output through the fully connected layer. The cross-entropy loss function is used to calculate the loss value, the backpropagation algorithm is used to adjust the model parameters, and the Adam optimizer is selected for optimization. (b) RNN model uses the LSTM method and consists of three layers with 64 neurons in each layer, yielding a final output of eight dimensions through the fully connected layer. Tanh is used as the activation function, and the cross entropy loss function and Adam optimizer are used to calculate the loss values and perform backpropagation optimization, respectively. The other construction and training processes are the same as those in the CNN training. The Raman signal data of each kind of bacteria include 1200 data points, which are used as convolution layer input of the CNN model and LSTM layer input of the long-term and shortterm memory model. Both models have two layers, but each layer of the CNN model includes a convolution layer and a pooling layer, while each layer of the LSTM model includes three gating units. Then, the model initializes the parameters of each layer randomly. Through the calculation of the model, the CNN and LSTM models obtain eight output values. Then, the eight values of the model are compared with the real species tag to obtain the error, and the parameters of the whole model are updated by the back propagation algorithm. Finally, through multiple rounds of training, the error between the eight output values of the CNN and LSTM models and the real species tag is minimized.

smoothing, and polynomial baseline fitting was performed to remove the fluorescence background. Then, the spectral data were normalized by using the min-max normalization method. The statistical packages of the $\mathrm{R}$ program were used throughout the process, including the "prospect", "baseline", and "clusterSim" packages. Finally, we removed the spectra with cosmic rays and truncated the remaining spectra to the region from 600 to $1800 \mathrm{~cm}^{-1}$. $44-46$
Model Architecture and Training Details. We built CNN and LSTM models with similar structures and layers and used the same data set for training and testing. The CNN model consists of two convolution layers, two pooling layers, and a fully connected layer, as shown in Figure 1a, with convolution layers containing 16 and 32 neurons. The output of eight dimensions is achieved through the maximum pooling layer and the fully connected layer. The RNN model uses the LSTM method, as shown in Figure $1 b$, and consists of three 


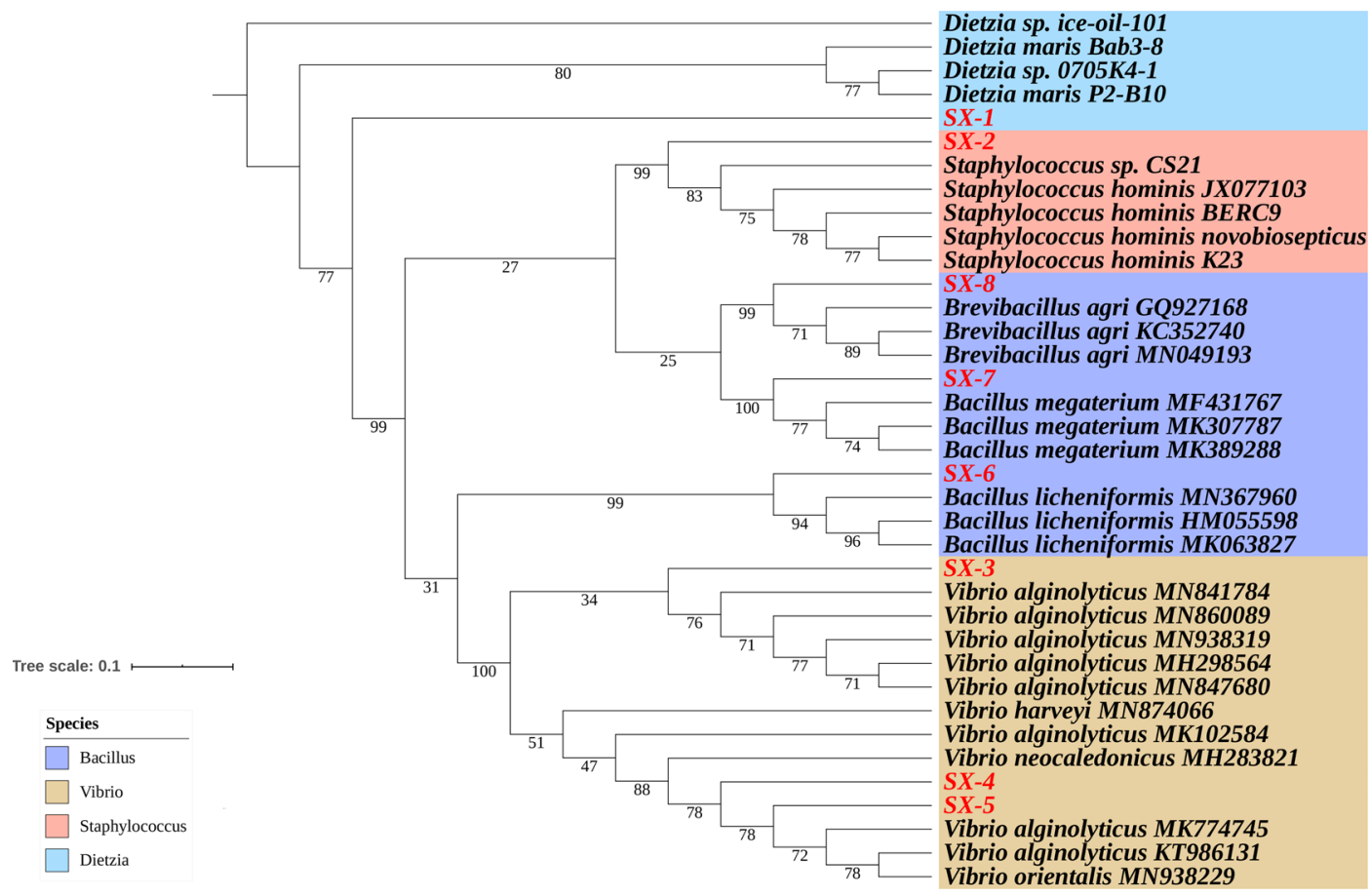

Figure 2. Phylogenetic tree of eight strains. SX-2 belongs to Staphylococcus, which has pathogenicity, and SX-3, SX-4, and SX-5 belong to Vibrio, which has potential pathogenicity.

layers with 64 neurons per layer, yielding a final output of eight dimensions through the fully connected layer. In training the $\mathrm{CNN}$ and RNN models, to ensure the conservativeness of the Raman spectral data features, we chose the signal data at every 20 lengths as the feature area; thus, we converted the data of 1 $\times 1200$ dimensions into data of $20 \times 60$ dimensions. ${ }^{47-51}$ To better define the trends of loss values and accuracy between the CNN model and the LSTM model during training, we superimposed the Raman data of three sets of parallel samples and fed the model for training using different data as the training set and testing set.

To further compare the classification accuracies of the CNN model and the LSTM model, we used the Raman data obtained from three sets of experiments that were independent of the data used for model training and performed crossvalidation on three parallel sets of samples. We subsequently tested the accuracy rate for each strain, and to reduce the error resulting from the uneven distribution of the training data, we used the mean of the sensitivity of all classes to avoid a classsize bias.

Exploration of the Raman Data by the Self-Designed Loss Function. The mapping of bacterial features in Raman data has been a difficult point in the field of analytical chemistry; therefore, we tried to explore the data by calculating the proximity of the models to the interbacterial affinities. With a conventionally trained model, although the desired classification results can be obtained, one cannot know what features the model learns from. When we test the sensitivity of the model input data by setting the requirements such that the results are the output based on what we know of the features, we can identify the bacterial features that the Raman data of the segment reflect. Since we constructed the phylogenetic tree based on sequencing results, it contained the numerical genetic distances between different strains, which is also considered nucleotide information. Therefore, according to these numer- ical results, we changed the label of the model training set so that it no longer had the traditional integer value but contained the distance distribution value. We chose the first strain as the control strain and set the label value to 1 . For the other strains, multiplication was used to set the label value according to the value standard of the distance from the first strain. This process is similar to changing the classification problem to a regression problem. However, the distance information in the tag is the relative distance rather than the absolute distance; thus, the loss function of the traditional regression model cannot be used for evaluation. Our focus is to make the model learn the phylogenetic distance between different strains and the relative gap between them. Thus, we constructed this loss function as follows

$$
\text { loss }=\operatorname{mean}\left(X / \bar{X}_{\text {label=«1» }}-Y\right)^{2}
$$

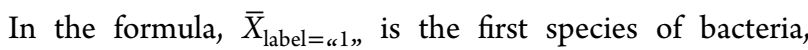
which we use as the reference bacteria. $X$ is the currently calculated bacterial species. By comparing them, the relative phylogenetic distance between the two species is obtained. $Y$ represents the true phylogenetic distance between the two species. By reducing the error between the calculated results and the real results, the purpose of training the model is realized. We selected SX-1, SX-3, SX-5, SX-6, and SX-8 as the strains for the training model and SX-1, SX-2, SX-4, and SX-7 as the strains for the testing model. Since the phylogenetic distance is a relative distance, SX-1 is required as a standard control strain in either the training or the test data set. SX-2 belongs to a different genus than the other strains, while SX-4 and SX-7 both belong to the same genus as the other strains but belong to different species; hence, the strain chosen is informative. After training the models, we found that they maintained good phylogenetic distance prediction compared to the untrained bacteria. We subsequently sorted 1200 numerical points of the Raman data per bacterial species into 

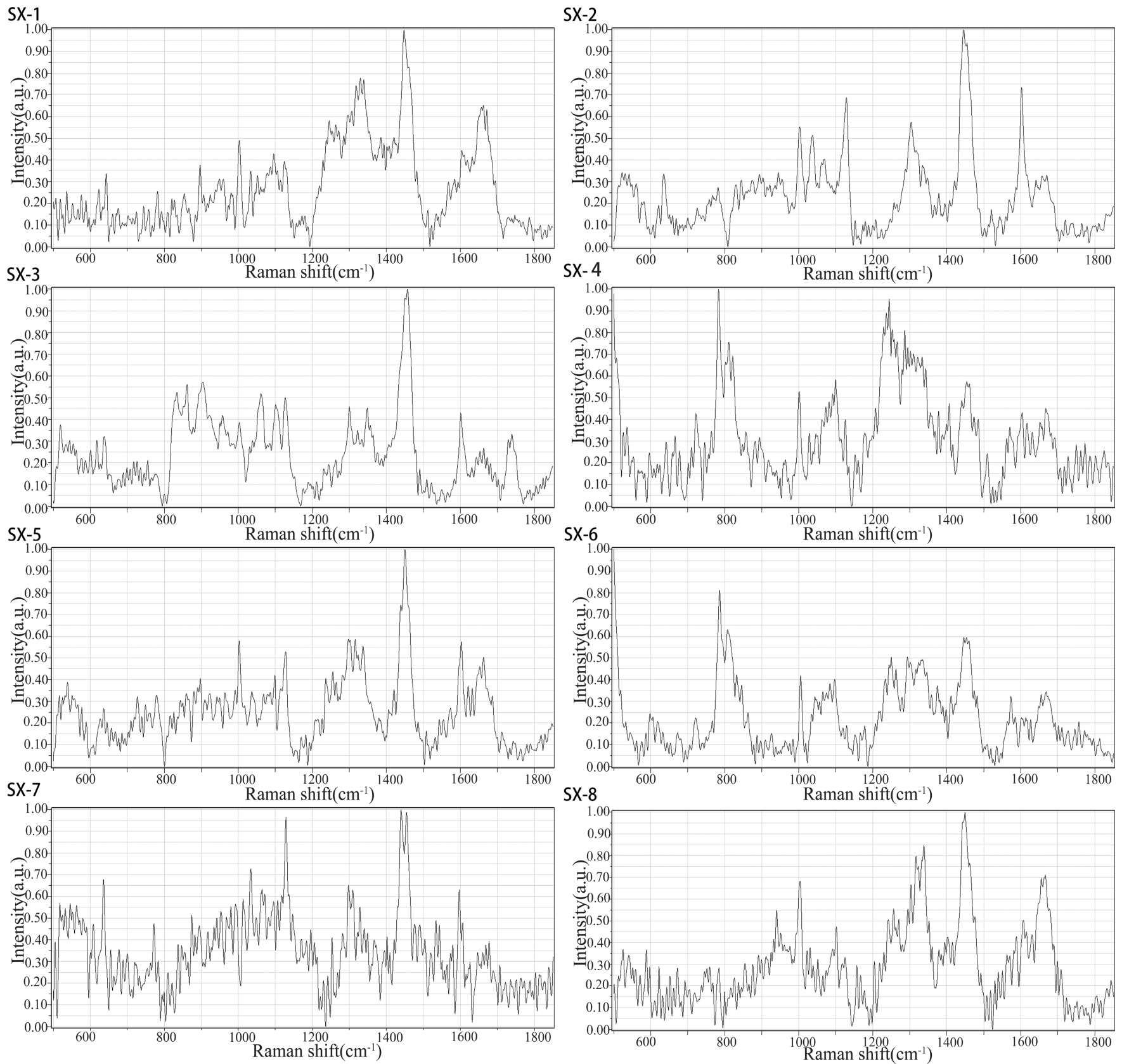

Figure 3. Raman spectra of the eight strains after preprocessing.

40 batches, each containing 30 data points. At the same time, we calculated the average of the Raman data for all bacteria, replacing the batches in the Raman data for each bacterium with the corresponding average numerical values. Finally, the processed Raman data were fed into the trained model, and the average variation in the loss value caused by replacing each set of batches was calculated. To highlight the change trend, we normalized the average change in the loss values and assumed that the patch location with the greatest change in the loss values was the region related to nucleic acid information. By reviewing the relevant literature, we can verify this hypothesis, and if the hypothesis holds, the model will be informative regarding the distribution of nucleic acid information in Raman data. At the same time, we chose other common loss functions for comparison. Because the classification label of the classification model can be only an integer value, we chose the mean squared error (MSE) loss of the regression model as the control model. To evaluate the effect of using MSE loss, we calculated the relative phylogenetic distance while training. Finally, we added two additional new bacteria to the test data to prove the applicability of the model.

\section{RESULTS}

Isolation and Identification of Intestinal Bacteria. Eight strains of bacteria were isolated from the intestine of $U$. unicinctus, and they were designated SX-1, SX-2, SX-3, SX-4, SX-5, SX-6, SX-7, and SX-8. All tested gene sequences of the eight strains were compared and analyzed through BLAST, as shown in Figure 2.

SX-2 belongs to Staphylococcus, which has pathogenicity. SX3, SX-4, and SX-5 belong to Vibrio, which has potential pathogenicity. Therefore, a reinfection test was carried out. ${ }^{52}$ 
a.

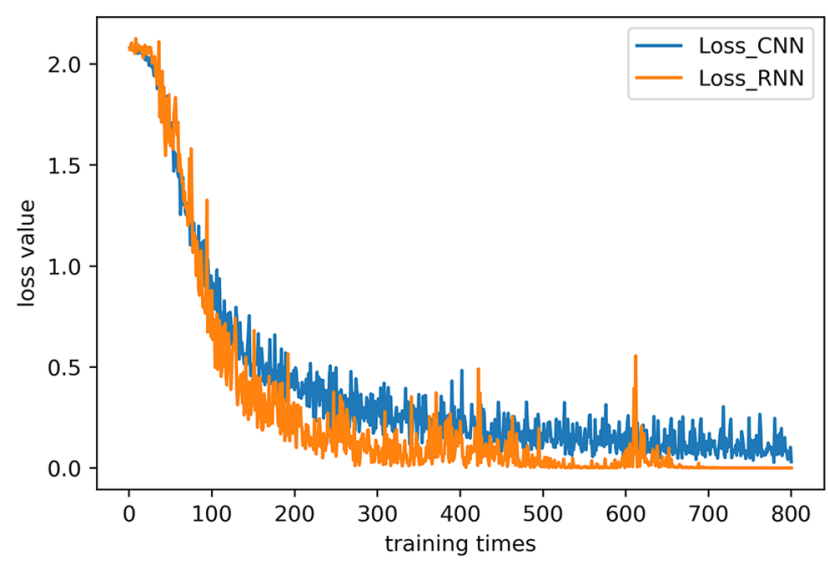

C.

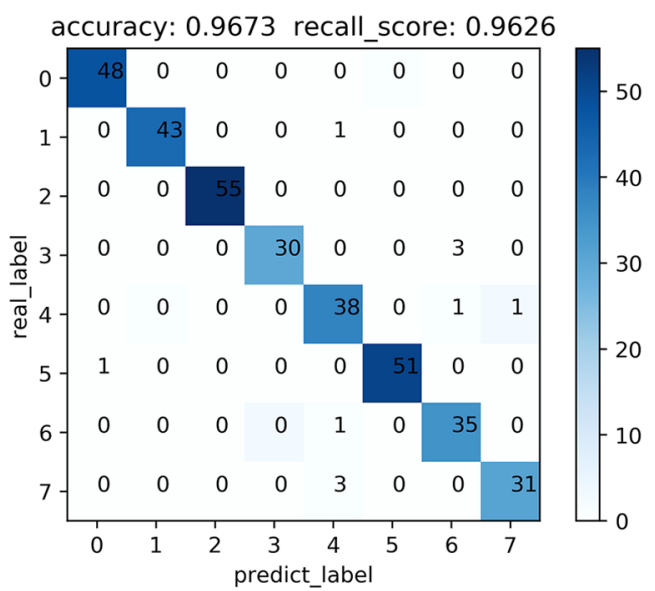

b.

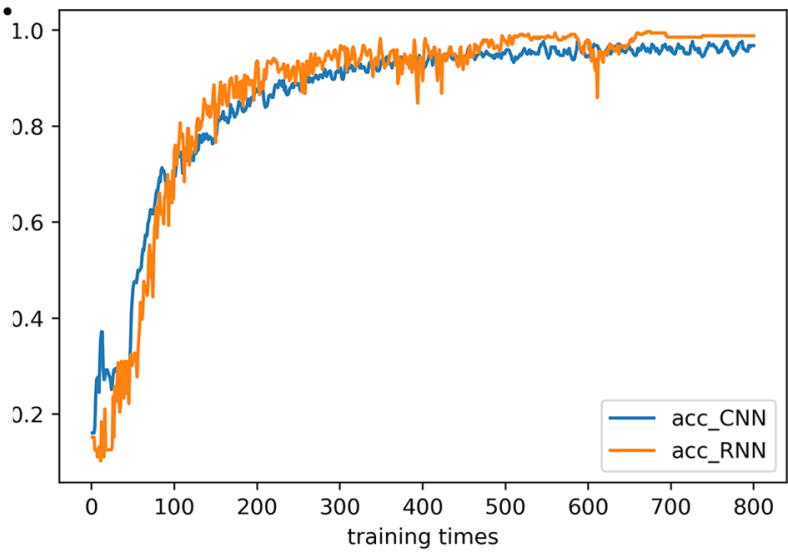

d.

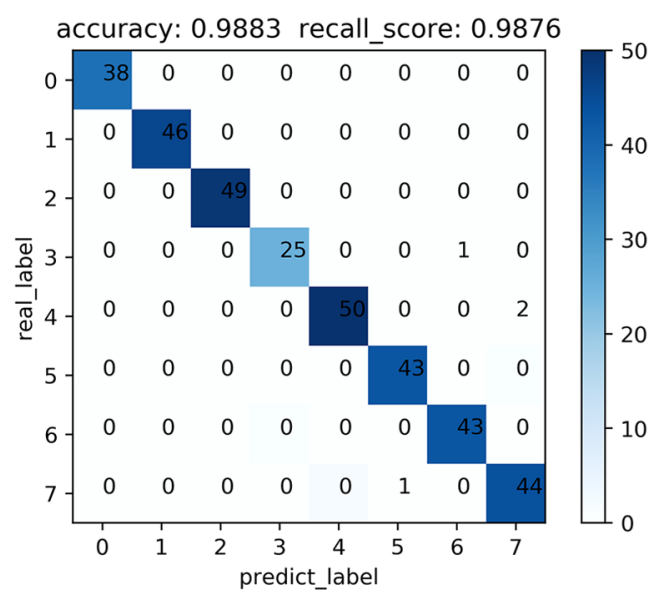

Figure 4. Training results. (a) Change in the loss value was recorded when the CNN and RNN models were trained. Clearly, although the two models dealt with the same Raman data, the training efficiency of the RNN model was higher, and the decrease in the loss value was lower. (b) Accuracy results of the CNN and RNN models for the test data set. (c,d) Final classification results of the two models. Clearly, the RNN model has a significantly improved accuracy rate compared to that of the CNN model.

Table 1. Cross-Validation Results of the CNN Model and the LSTM Model

\begin{tabular}{|c|c|c|c|c|c|c|c|c|c|}
\hline \multicolumn{10}{|c|}{$\begin{array}{c}\text { CNN results } \\
\text { mean accuracy: } 0.89\end{array}$} \\
\hline & SX-1 & SX-2 & SX-3 & SX-4 & SX-5 & SX-6 & SX-7 & SX-8 & mean \\
\hline sample1 & 0.9 & 0.9 & 0.9 & 0.96 & 0.9 & 0.9 & 0.9 & 0.9 & 0.9 \\
\hline sample2 & 0.92 & 0.92 & 0.92 & 0.9 & 0.9 & 0.92 & 0.92 & 0.92 & 0.91 \\
\hline sample3 & 0.86 & 0.86 & 0.86 & 0.90 & 0.82 & 0.86 & 0.86 & 0.86 & 0.86 \\
\hline mean & 0.89 & 0.89 & 0.89 & 0.92 & 0.87 & 0.89 & 0.89 & 0.89 & 0.89 \\
\hline \multirow{2}{*}{\multicolumn{10}{|c|}{$\begin{array}{l}\text { LSTM results } \\
\text { mean accuracy: } 0.94\end{array}$}} \\
\hline & & & & & & & & & \\
\hline & SX-1 & SX-2 & SX-3 & SX-4 & SX-5 & SX-6 & SX-7 & SX-8 & mean \\
\hline sample1 & 1.0 & 1.0 & 1.0 & 1.0 & 1.0 & 1.0 & 1.0 & 1.0 & 1.0 \\
\hline sample2 & 1.0 & 1.0 & 1.0 & 1.0 & 1.0 & 1.0 & 1.0 & 1.0 & 1.0 \\
\hline sample3 & 0.84 & 0.84 & 0.84 & 0.90 & 0.8 & 0.84 & 0.84 & 0.84 & 0.84 \\
\hline mean & 0.94 & 0.94 & 0.94 & 0.96 & 0.93 & 0.94 & 0.94 & 0.94 & 0.94 \\
\hline
\end{tabular}

Manual Infection Resumption Test. U. unicinctus showed similar symptoms after these three Vibrio strains were added. The day after manual infection resumption, the $U$. unicinctus larvae began to drill themselves out from the sand with weakening activity and the appearance of red coloration on the surface. The symptoms were the same as those of the diseased larvae from which pure bacteria were extracted for the first time. On the third day, the mortality reached $40 \%$, and on the fifth day, all of the U. unicinctus larvae were dead. Thus, we determined that all three Vibrio strains were pathogenic.

Raman Spectroscopy Results. The Raman spectra of the eight strains are shown in Figure 3. We randomly selected 50 spectra for each strain to compare the differences among them. 
a.

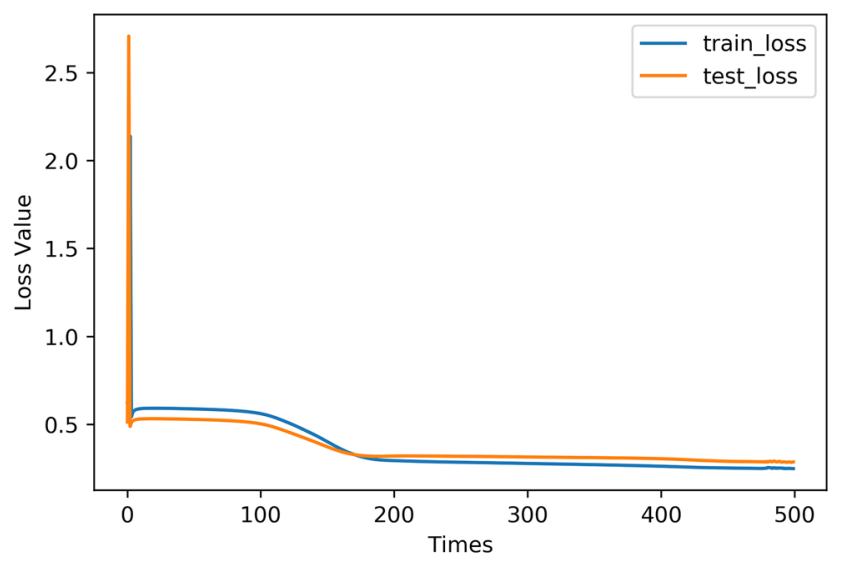

c.

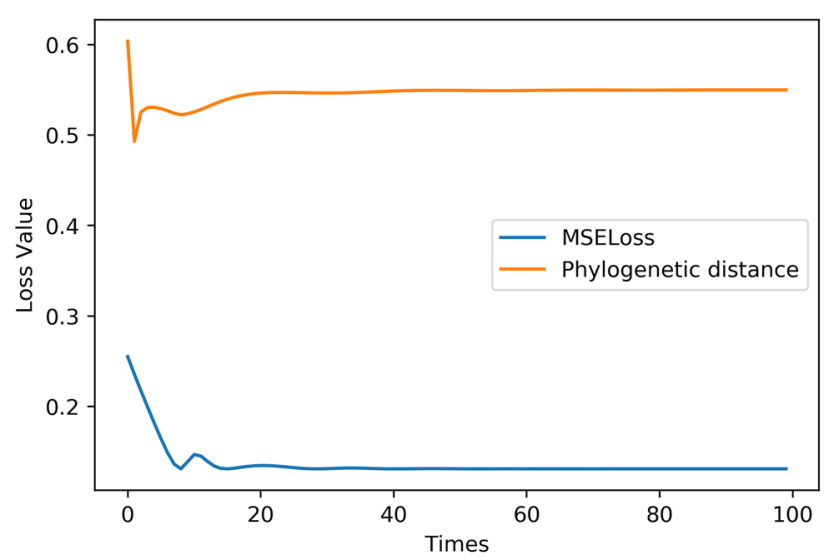

$\boldsymbol{e}$.

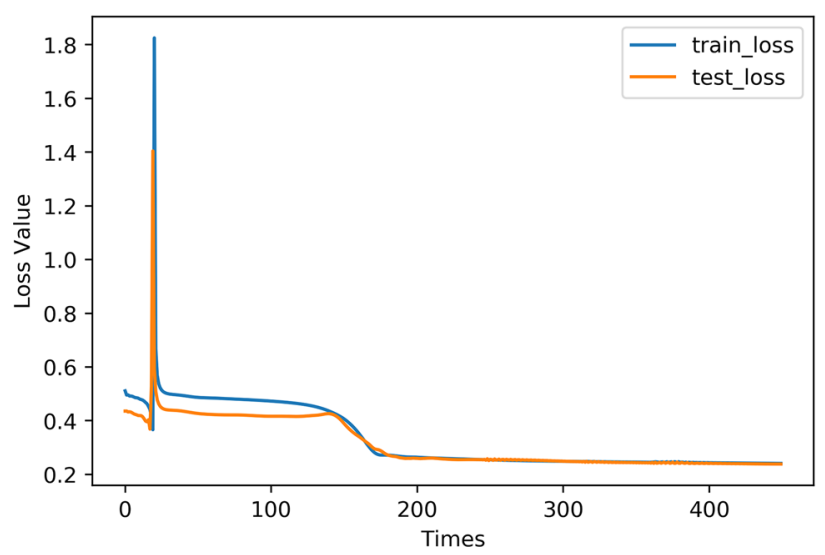

b.

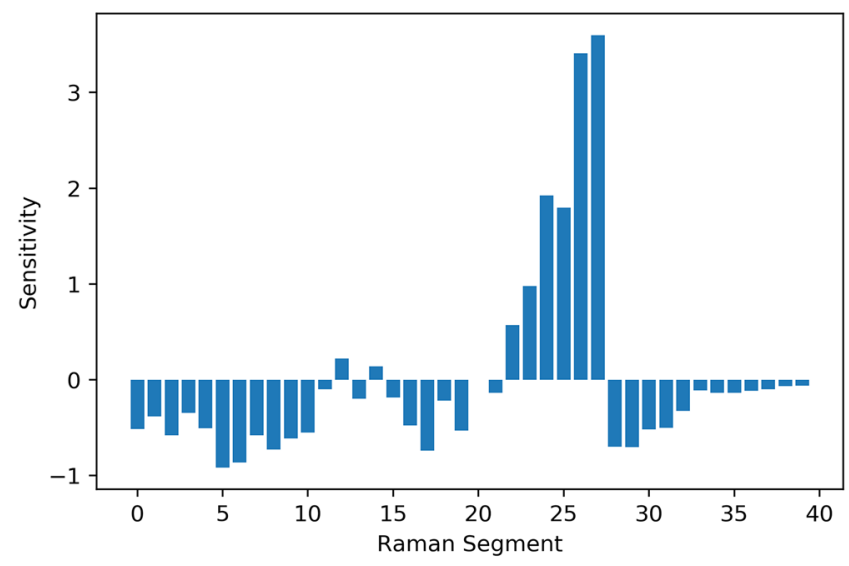

d.

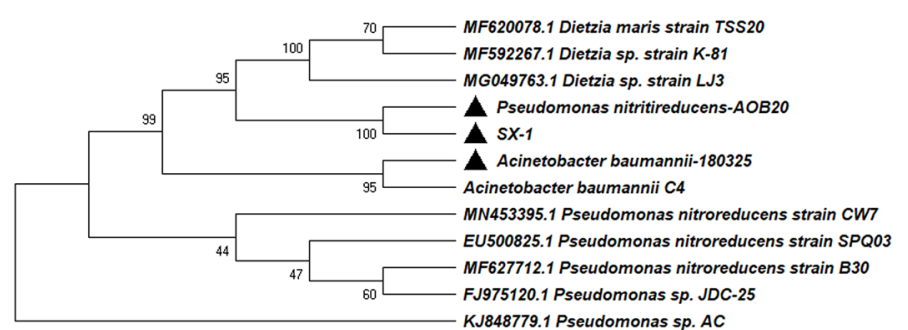

$f$.

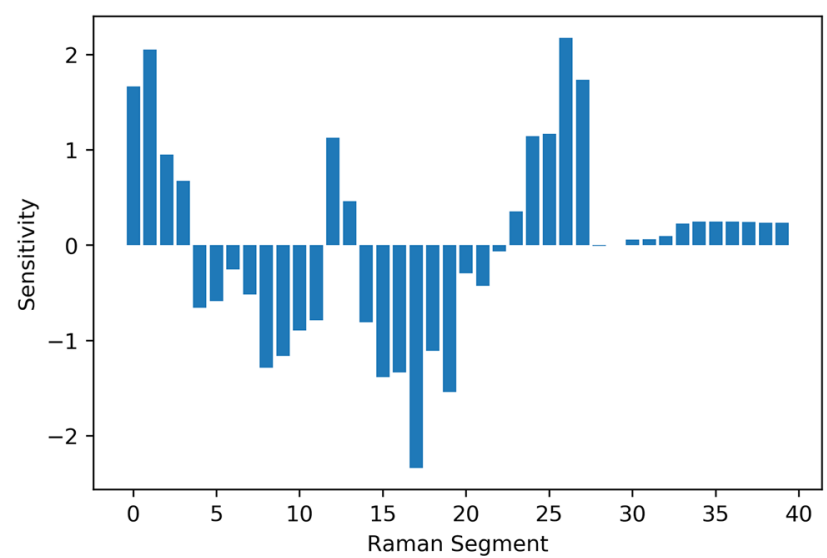

Figure 5. Raman data analysis results.

As shown in Figure 3, it was difficult to identify the feature information directly.

The average Raman data of the eight strains after preprocessing, which included background removal and normalization, are shown in the figure.

Novel Methods for Bacterial Classification from Raman Spectra. As shown in Figure 4a,b, during training of the CNN and RNN, the loss value of the RNN decreased the fastest, and the final loss value was lower than that of the $\mathrm{CNN}$, which proves that the $\mathrm{RNN}$ training process is faster and more accurate than that of the $\mathrm{CNN}$. The training results of the last two models on the test data set also prove that the RNN has more advantages than the $\mathrm{CNN}$ in processing Raman data. As shown in Figure 4c,d, the accuracy and recall rate of the RNN were both higher than those of the CNN model. As shown in Table 1, the classification accuracy of the LSTM model was greater than that of the CNN model.

The table shows the classification accuracy of the CNN model and LSTM model for these eight kinds of bacteria. To reduce the error caused by the inconsistent size of the data set, we take the average of the classification accuracy of the eight bacteria as the final accuracy of the model. The final accuracies 
of the CNN model and LSTM model are 0.89 and 0.94 , respectively.

Raman Data Analysis Results. We chose the LSTM framework as a fully trained model for testing; the sensitivity test results are shown in Figure 5a,b. The models maintained good phylogenetic distance prediction from the untrained bacteria, and the maximum was obtained for segment batch 26 . Variation in this segment contributed the greatest effect on kinship variation among the eight bacterial species, and we, therefore, predict that this Raman segment reflects the nucleic acid characteristics of the bacteria.

Figure 5a shows the changes in the loss value of the training data set and test data set during model training. Figure $5 \mathrm{~b}$ shows the sensitivity of different Raman positions to the model. The Raman signal data contained a total of 1200 data points. We selected every 30 data points as a segment, so there were a total of 40 segments. Then, we compared the sensitivity of these 40 segments. The highest sensitivity was obtained for segments $26-27$. Figure $5 \mathrm{c}$ shows the changes in the predicted relative phylogenetic distances while training the model with the MSE loss function. The phylogenetic tree of the two newly added bacteria and the relative phylogenetic distance with respect to SX-1 is displayed in Figure 5d. Figure 5e,f shows the changes in the loss value and the final sensitivity of the model after the two new bacteria were added.

Figure $5 \mathrm{c}$ shows that although the model converged when the MSE loss function was used, the relative phylogenetic distance predicted by the model was very different, remaining almost the same as that before training. However, the model trained by the loss function proposed in this study could greatly reduce the prediction gap. We constructed a phylogenetic tree of the two newly acquired bacteria and obtained the relative developmental distance, as shown in Figure 5d. Then, the Raman data of the two bacteria were added to the test data set for prediction. As shown in Figure $5 e, f$, the model clearly still showed good convergence for the new strain. At the same time, according to the sensitivity of the model, the position associated with the nucleic acid information was also batches 26-27. Therefore, we speculated that the region of batches $26-27$ is a nucleic acid-related region based on the sensitivity of the two models. Previously, two characteristic positions which might represent some comparative conservation among strains were found by comparing the differences among the Raman spectra of different strains. One of these was at position 1400 relative to the spectral data, which was also present in our batch 26; this fit was even more indicative of the possibility that this study found a nucleic acid signature at batch 26 . According to previous studies, ${ }^{49,53}$ the position of batches $26-27$ was determined to contain nucleic acid information. Therefore, this finding suggests that the model is of great reference value.

\section{DISCUSSION}

In this study, eight strains of intestinal pathogens were isolated from aquacultured $U$. unicinctus larvae. To date, few studies have emerged on the diseases of $U$. unicinctus larvae caused by microorganisms; therefore, research on intestinal pathogens is conducive to the sustainable development of $U$. unicinctus farming and is of practical guiding significance for healthy aquaculture of $U$. unicinctus.

We chose more suitable deep learning techniques combined with Raman spectroscopy to identify gut-associated pathogens; deep learning methods have already been widely used in
Raman data classification. ${ }^{54-59}$ Compared with other molecular biological and culture-free methods, such as single-cell sequencing ${ }^{60}$ and fluorescence spectroscopy, Raman spectroscopy is easily generalized to new strains because it does not require separate label settings. For the analysis methods, LSTM networks can classify different species faster and better than other convolutional network methods. ${ }^{28}$ When combined with deep learning networks, the Raman deep learning methods presented here can rapidly scan and identify every bacterium from a diseased sample in a culture-free manner in only a few seconds. This technique will allow us to perform accurate and effective treatment of pathogenic bacterial infections within hours. ${ }^{61,62}$ However, as shown in our research, $\mathrm{CNN}$ models can learn only the information between the feature nodes in Raman data. The RNN network is able to fuse the information of the front and back nodes well and thus learn more from a limited data set. When we input the Raman data into a CNN, 1200 data points pass through a convolution layer and a pooling layer for feature extraction. Then, the input data are dimensionally reduced, and the neural network is trained in the fully connected layer. Finally, the output is given in eight dimensions, and the location of the maximum output value is selected as the label value of the strain. The LSTM neural network is an improvement on the CNN and RNN models. Three gating mechanisms are added in each layer, namely, the update gate, forget gate, and output gate. The forget gate determines how much of the state of the previous moment remains in the current moment. The input gate determines how much input at the current time is saved to the state. The output gate controls how much information in the state is used as the output. It is well known that certain characteristics of bacteria, such as the nucleic acid information, in the Raman spectrum appear as not only a peak but also a distribution across different regions of the entire Raman data set. Therefore, when we feed the Raman data into the LSTM network, the model can comprehensively evaluate the feature information distributed in the whole Raman full-length segment by superimposing the retained feature information onto the feature information at this time, while the traditional $\mathrm{CNN}$ can select only some of this feature information as the reference standard. Then, the model feeds the superimposed comprehensive feature information into the fully connected layer to achieve more effective and accurate classification. Therefore, regarding the efficient and full utilization of resources, this method has tremendous potential to be developed as a tool for pathogen identification, and LSTM networks will contribute greatly to biomedical and sustainable development research in the future.

Traditional loss functions are used in classification models and regression models, and the loss function of classification models cannot calculate the class label of a noninteger value. Although the loss functions of regression models can train the model, the training results are more inclined toward absolute position information than phylogenetic distance information. Therefore, the final standard to evaluate the training quality of regression models is still using the loss model constructed in this study to calculate the prediction results. We also performed a preliminary analysis of how the Raman data reflect the strain, and although the sensitivity was highest in this segment, we believe that the peak in the Raman data should be a mixed peak with a variety of features in common and with only the most obvious of those reflected in the characteristics of the nucleic acids in this segment. Accurate 
analysis of Raman data is very important in Raman studies, and many novel methods have been endeavored to achieve this goal. The present methods of exploring the mechanism behind the deep learning model offer a valuable perspective regarding the accurate analysis of Raman data in the field of analytical chemistry.

\section{AUTHOR INFORMATION}

\section{Corresponding Authors}

Yu Vincent Fu - State Key Laboratory of Microbial Resources, Institute of Microbiology, Chinese Academy of Sciences, Beijing 100101, P. R. China; University of the Chinese Academy of Sciences, Beijing 100049, P. R. China; (1) orcid.org/0000-0001-8251-2306; Email: fuyu@im.ac.cn

Fanghua Liu - Key Laboratory of Coastal Biology and Biological Resources Utilization, CAS Key Laboratory of Coastal Environmental Processes and Ecological Remediation, Yantai Institute of Coastal Zone Research, Chinese Academy of Sciences, Yantai 264003, P. R. China; National-Regional Joint Engineering Research Center for Soil Pollution Control and Remediation in South China, Guangdong Key Laboratory of Integrated Agro-environmental Pollution Control and Management, Institute of Eco-environmental and Soil Sciences, Guangdong Academy of Sciences, Guangzhou 510650, P. R. China; ○orcid.org/0000-0002-1934-3674; Email: fhliu@yic.ac.cn

\section{Authors}

Shixiang Yu - Key Laboratory of Coastal Biology and Biological Resources Utilization, CAS Key Laboratory of Coastal Environmental Processes and Ecological Remediation, Yantai Institute of Coastal Zone Research, Chinese Academy of Sciences, Yantai 264003, P. R. China; University of the Chinese Academy of Sciences, Beijing 100049, P. R. China

Xin Li - Key Laboratory of Coastal Biology and Biological Resources Utilization, CAS Key Laboratory of Coastal Environmental Processes and Ecological Remediation, Yantai Institute of Coastal Zone Research, Chinese Academy of Sciences, Yantai 264003, P. R. China; University of the Chinese Academy of Sciences, Beijing 100049, P. R. China

Weilai Lu - State Key Laboratory of Microbial Resources, Institute of Microbiology, Chinese Academy of Sciences, Beijing 100101, P. R. China; University of the Chinese Academy of Sciences, Beijing 100049, P. R. China

Hanfei Li - State Key Laboratory of Microbial Resources, Institute of Microbiology, Chinese Academy of Sciences, Beijing 100101, P. R. China; University of the Chinese Academy of Sciences, Beijing 100049, P. R. China

Complete contact information is available at:

https://pubs.acs.org/10.1021/acs.analchem.1c00431

\section{Author Contributions}

All authors contributed extensively to the work presented in this paper. S.Y.: investigation, formal analysis, writing-original draft, writing-review and editing. W.L.: collection and processing of supplementary data. H.L.: resources. X.L.: resources. Y.V.F.: conceptualization. F.L.: ronceptualization, writing-original draft, writing-review and editing.

Notes

The authors declare no competing financial interest. Relevant data and code have been uploaded to GitHub https://github.com/ArthurWillYu/CNN-RNN-Raman.

\section{ACKNOWLEDGMENTS}

We thank Prof. Feng Liu and Prof. Yuan Ji of China Agricultural University for providing Urechis unicinctus and helping with breeding. This research was financially supported by the National Key Research and Development Program of China (grant no. 2020YFA0907300), the National Natural Science Foundation of China (grant nos. U20A20109 and 91751103), the Guangdong Foundation for Program of Science and Technology Research (grant nos. 2020B1111530002 and 2020B1212060048), the GDAS' Project of Science and Technology Development (2019GDASYL-0102003 and 2019GDASYL-0102005), the Pearl River Talent Recruitment Program (2019QN01L735) of Guangdong Province, the Chinese Academy of Sciences (GQRC-1918 ), and the Senior User Project of RV KEXUE (KEXUE2019GZ05).

\section{REFERENCES}

(1) Qin, Z.; Zhang, Y.; Mu, H.; Zhang, Z.; Qiu, J. W. Proteomics 2018, 18, No. e1800107.

(2) Oh, H. Y.; Kim, C.-H.; Go, H.-J.; Park, N. G. Fish Shellfish Immunol. 2018, 79, 351-362.

(3) Detweiler, C. S. Trends Microbiol. 2017, 25, 169-170.

(4) Wang, Y.; Shi, T.; Huang, G.; Gong, J. Microb. Environ. 2018, 33, 290-300.

(5) Zhang, L.; Liu, X.; Liu, J.; Zhang, Z. PLoS One 2013, 8, No. e81885.

(6) Wu, J.-B.; Lin, M.-L.; Cong, X.; Liu, H.-N.; Tan, P.-H. Chem. Soc. Rev. 2018, 47, 1822-1873.

(7) Langer, J.; Jimenez de Aberasturi, D.; Aizpurua, J.; AlvarezPuebla, R. A.; Auguié, B.; Baumberg, J. J.; Bazan, G. C.; Bell, S. E. J.; Boisen, A.; Brolo, A. G.; Choo, J.; Cialla-May, D.; Deckert, V.; Fabris, L.; Faulds, K.; García de Abajo, F. J.; Goodacre, R.; Graham, D.; Haes, A. J.; Haynes, C. L.; Huck, C.; Itoh, T.; Käll, M.; Kneipp, J.; Kotov, N. A.; Kuang, H.; Le Ru, E. C.; Lee, H. K.; Li, J.-F.; Ling, X. Y.; Maier, S. A.; Mayerhöfer, T.; Moskovits, M.; Murakoshi, K.; Nam, J.-M.; Nie, S.; Ozaki, Y.; Pastoriza-Santos, I.; Perez-Juste, J.; Popp, J.; Pucci, A.; Reich, S.; Ren, B.; Schatz, G. C.; Shegai, T.; Schlücker, S.; Tay, L.-L.; Thomas, K. G.; Tian, Z.-Q.; Van Duyne, R. P.; Vo-Dinh, T.; Wang, Y.; Willets, K. A.; Xu, C.; Xu, H.; Xu, Y.; Yamamoto, Y. S.; Zhao, B.; Liz-Marzán, L. M. ACS Nano 2020, 14, 28-117.

(8) Fan, M.; Andrade, G. F. S.; Brolo, A. G. Anal. Chim. Acta 2020, 1097, 1-29.

(9) Pilot, R.; Signorini, R.; Durante, C.; Orian, L.; Bhamidipati, M.; Fabris, L. Biosensors 2019, 9, 57.

(10) Reguera, J.; Langer, J.; Jiménez de Aberasturi, D.; Liz-Marzán, L. M. Chem. Soc. Rev. 2017, 46, 3866-3885.

(11) Li, J.-F.; Zhang, Y.-J.; Ding, S.-Y.; Panneerselvam, R.; Tian, Z.Q. Chem. Rev. 2017, 117, 5002-5069.

(12) Ding, S.-Y.; You, E.-M.; Tian, Z.-Q.; Moskovits, M. Chem. Soc. Rev. 2017, 46, 4042-4076.

(13) Knebl, A.; Domes, R.; Yan, D.; Popp, J.; Trumbore, S.; Frosch, T. Anal. Chem. 2019, 91, 7562-7569.

(14) Bocklitz, T. W.; Crecelius, A. C.; Matthäus, C.; Tarcea, N.; von Eggeling, F.; Schmitt, M.; Schubert, U. S.; Popp, J. Anal. Chem. 2013, $85,10829-10834$.

(15) Ryabchykov, O.; Popp, J.; Bocklitz, T. Front. Chem. 2018, 6, 257.

(16) Liu, Y.; Zhou, H.; Hu, Z.; Yu, G.; Yang, D.; Zhao, J. Biosens. Bioelectron. 2017, 94, 131-140.

(17) Cui, L.; Yang, K.; Li, H.-Z.; Zhang, H.; Su, J.-Q.; Paraskevaidi, M.; Martin, F. L.; Ren, B.; Zhu, Y.-G. Anal. Chem. 2018, 90, 50825089.

(18) Pang, Y.; Wan, N.; Shi, L.; Wang, C.; Sun, Z.; Xiao, R.; Wang, S. Anal. Chim. Acta 2019, 1077, 288-296. 
(19) Song, Y.; Kaster, A.-K.; Vollmers, J.; Song, Y.; Davison, P. A.; Frentrup, M.; Preston, G. M.; Thompson, I. P.; Murrell, J. C.; Yin, H.; Hunter, C. N.; Huang, W. E. Microb. Biotechnol. 2017, 10, 125-137.

(20) Tong, D.; Chen, C.; Zhang, J.; Lv, G.; Zheng, X.; Zhang, Z.; Lv, X. Photodiagnosis Photodyn. Ther. 2019, 28, 248-252.

(21) Novelli-Rousseau, A.; Espagnon, I.; Filiputti, D.; Gal, O.; Douet, A.; Mallard, F.; Josso, Q. Sci. Rep. 2018, 8, 3957.

(22) Lussier, F.; Missirlis, D.; Spatz, J. P.; Masson, J. F. ACS Nano 2019, 13, 1403-1411.

(23) Boardman, A. K.; Wong, W. S.; Premasiri, W. R.; Ziegler, L. D.; Lee, J. C.; Miljkovic, M.; Klapperich, C. M.; Sharon, A.; Sauer-Budge, A. F. Anal. Chem. 2016, 88, 8026-8035.

(24) Suzuki, Y.; Kobayashi, K.; Wakisaka, Y.; Deng, D.; Tanaka, S.; Huang, C.-J.; Lei, C.; Sun, C.-W.; Liu, H.; Fujiwaki, Y.; Lee, S.; Isozaki, A.; Kasai, Y.; Hayakawa, T.; Sakuma, S.; Arai, F.; Koizumi, K.; Tezuka, H.; Inaba, M.; Hiraki, K.; Ito, T.; Hase, M.; Matsusaka, S.; Shiba, K.; Suga, K.; Nishikawa, M.; Jona, M.; Yatomi, Y.; Yalikun, Y.; Tanaka, Y.; Sugimura, T.; Nitta, N.; Goda, K.; Ozeki, Y. Proc. Natl. Acad. Sci. U. S. A. 2019, 116, 15842-15848.

(25) Raumviboonsuk, P.; Krause, J.; Chotcomwongse, P.; Sayres, R.; Raman, R.; Widner, K.; Campana, B. J. L.; Phene, S.; Hemarat, K.; Tadarati, M.; Silpa-Archa, S.; Limwattanayingyong, J.; Rao, C.; Kuruvilla, O.; Jung, J.; Tan, J.; Orprayoon, S.; Kangwanwongpaisan, C.; Sukumalpaiboon, R.; Luengchaichawang, C.; Fuangkaew, J.; Kongsap, P.; Chualinpha, L.; Saree, S.; Kawinpanitan, S.; Mitvongsa, K.; Lawanasakol, S.; Thepchatri, C.; Wongpichedchai, L.; Corrado, G. S.; Peng, L.; Webster, D. R. NPJ Digit. Med. 2019, 2, 25.

(26) Hollon, T. C.; Pandian, B.; Adapa, A. R.; Urias, E.; Save, A. V.; Khalsa, S. S. S.; Eichberg, D. G.; D'Amico, R. S.; Farooq, Z. U.; Lewis, S.; Petridis, P. D.; Marie, T.; Shah, A. H.; Garton, H. J. L.; Maher, C. O.; Heth, J. A.; McKean, E. L.; Sullivan, S. E.; Hervey-Jumper, S. L.; Patil, P. G.; Thompson, B. G.; Sagher, O.; McKhann, G. M., 2nd; Komotar, R. J.; Ivan, M. E.; Snuderl, M.; Otten, M. L.; Johnson, T. D.; Sisti, M. B.; Bruce, J. N.; Muraszko, K. M.; Trautman, J.; Freudiger, C. W.; Canoll, P.; Lee, H.; Camelo-Piragua, S.; Orringer, D. A. Nat. Med. 2020, 26, 52-58.

(27) Singh, R.; Kalra, M. K.; Nitiwarangkul, C.; Patti, J. A.; Homayounieh, F.; Padole, A.; Rao, P.; Putha, P.; Muse, V. V.; Sharma, A.; Digumarthy, S. R. PLoS One 2018, 13, No. e0204155.

(28) Fan, X.; Ming, W.; Zeng, H.; Zhang, Z.; Lu, H. Analyst 2019, 144, 1789-1798.

(29) González-Durruthy, M.; Alberici, L. C.; Curti, C.; Naal, Z.; Atique-Sawazaki, D. T.; Vázquez-Naya, J. M.; González-Díaz, H.; Munteanu, C. R. J. Chem. Inf. Model. 2017, 57, 1029-1044.

(30) Tadesse, L. F.; Safir, F.; Ho, C.-S.; Hasbach, X.; Khuri-Yakub, B.; Jeffrey, S. S.; Saleh, A. A. E.; Dionne, J. J. Chem. Phys. 2020, 152, 240902.

(31) Zhang, L.; Wu, Y.; Zheng, B.; Su, L.; Chen, Y.; Ma, S.; Hu, Q.; Zou, X.; Yao, L.; Yang, Y.; Chen, L.; Mao, Y.; Chen, Y.; Ji, M. Theranostics 2019, 9, 2541-2554.

(32) Weng, S.; Xu, X.; Li, J.; Wong, S. T. C. J. Biomed. Opt. 2017, 22, $1-10$.

(33) Tadesse, L. F.; Ho, C.-S.; Chen, D.-H.; Arami, H.; Banaei, N.; Gambhir, S. S.; Jeffrey, S. S.; Saleh, A. A. E.; Dionne, J. Nano Lett. 2020, 20, 7655-7661.

(34) Ting, D. S. W.; Pasquale, L. R.; Peng, L.; Campbell, J. P.; Lee, A. Y.; Raman, R.; Tan, G. S. W.; Schmetterer, L.; Keane, P. A.; Wong, T. Y. Br. J. Ophthalmol. 2019, 103, 167-175.

(35) Krauß, S. D.; Roy, R.; Yosef, H. K.; Lechtonen, T.; El-Mashtoly, S. F.; Gerwert, K.; Mosig, A. J. Biophot. 2018, 11, No. e201800022.

(36) You, J. M.; Guo, J.; Li, Z.; Yang, Q.; Duan, Y. Y.; Guo, X. L.; Huang, D. Y.; Zou, Z. L.; Guo, H. J. China J. Chin. Mater. Med. 2019, 44, 3954-3959.

(37) Schulte, L. N.; Schweinlin, M.; Westermann, A. J.; Janga, H.; Santos, S. C.; Appenzeller, S.; Walles, H.; Vogel, J.; Metzger, M. mBio 2020, 11, No. e03348-19.

(38) Pannkuk, E. L.; Risch, T. S.; Savary, B. J. PLoS One 2015, 10, No. e0120508.
(39) Zhang, Z.; Yang, C. M.; Li, Y.; Sui, C.; Liang, L.; Chu, Q. L. China J. Chin. Mater. Med. 2013, 38, 1500-1503.

(40) Tao, H.-P.; Shen, Z.-Y.; Zhu, F.; Xu, X.-F.; Tang, X.-D.; Xu, L. Curr. Microbiol. 2011, 62, 876-883.

(41) Supabandhu, J.; Fisher, M. C.; Mendoza, L.; Vanittanakom, N. Med. Mycol. 2008, 46, 41-52.

(42) Bagaglio, S.; Bianchi, G.; Danise, A.; Porrino, L.; Uberti-Foppa, C.; Lazzarin, A.; Castagna, A.; Morsica, G. Infection 2011, 39, 121126.

(43) Parachalil, D. R.; Brankin, B.; McIntyre, J.; Byrne, H. J. Analyst 2018, 143, 5987-5998.

(44) Saviello, D.; Trabace, M.; Alyami, A.; Mirabile, A.; Baglioni, P.; Giorgi, R.; Iacopino, D. Front. Chem. 2019, 7, 727.

(45) Lu, W.; Chen, X.; Wang, L.; Li, H.; Fu, Y. V. Anal. Chem. 2020, 92, 6288-6296.

(46) Li, J.; Wang, C.; Shi, L.; Shao, L.; Fu, P.; Wang, K.; Xiao, R.; Wang, S.; Gu, B. Mikrochim. Acta 2019, 186, 475.

(47) Ho, C.-S.; Jean, N.; Hogan, C. A.; Blackmon, L.; Jeffrey, S. S.; Holodniy, M.; Banaei, N.; Saleh, A. A. E.; Ermon, S.; Dionne, J. Nat. Commun. 2019, 10, 4927.

(48) Berisha, S.; Lotfollahi, M.; Jahanipour, J.; Gurcan, I.; Walsh, M.; Bhargava, R.; Van Nguyen, H.; Mayerich, D. Analyst 2019, 144, $1642-1653$

(49) Gurbani, S. S.; Schreibmann, E.; Maudsley, A. A.; Cordova, J. S.; Soher, B. J.; Poptani, H.; Verma, G.; Barker, P. B.; Shim, H.; Cooper, L. A. D. Magn. Reson. Med. 2018, 80, 1765-1775.

(50) Fulton, L. V.; Dolezel, D.; Harrop, J.; Yan, Y.; Fulton, C. P. Brain Sci. 2019, 9, 212.

(51) He, F.; Liu, T.; Tao, D. IEEE Trans. Neural. Netw. Learn. Syst. 2020, 31, 5349.

(52) Chen, X.; Tang, M.; Liu, Y.; Huang, J.; Liu, Z.; Tian, H.; Zheng, Y.; de la Chapelle, M. L.; Zhang, Y.; Fu, W. Mikrochim. Acta 2019, $186,102$.

(53) Lorenz, B.; Wichmann, C.; Stöckel, S.; Rösch, P.; Popp, J. Trends Microbiol. 2017, 25, 413-424.

(54) Liu, J.; Osadchy, M.; Ashton, L.; Foster, M.; Solomon, C. J.; Gibson, S. J. Analyst 2017, 142, 4067-4074.

(55) Acquarelli, J.; van Laarhoven, T.; Gerretzen, J.; Tran, T. N.; Buydens, L. M. C.; Marchiori, E. Anal. Chim. Acta 2017, 954, 22-31.

(56) Fukuhara, M.; Fujiwara, K.; Maruyama, Y.; Itoh, H. Anal. Chim. Acta 2019, 1087, 11-19.

(57) He, H.; Xu, M.; Zong, C.; Zheng, P.; Luo, L.; Wang, L.; Ren, B. Anal. Chem. 2019, 91, 7070-7077.

(58) Rodner, E.; Bocklitz, T.; von Eggeling, F.; Ernst, G.; Chernavskaia, O.; Popp, J.; Denzler, J.; Guntinas-Lichius, O. Head Neck 2019, 41, 116-121.

(59) Guselnikova, O.; Trelin, A.; Skvortsova, A.; Ulbrich, P.; Postnikov, P.; Pershina, A.; Sykora, D.; Svorcik, V.; Lyutakov, O. Biosens. Bioelectron. 2019, 145, 111718.

(60) Wang, Y.; Navin, N. E. Mol. Cell 2015, 58, 598-609.

(61) Chen, Y.; Premasiri, W. R.; Ziegler, L. D. Sci. Rep. 2018, 8, 5163.

(62) Manifold, B.; Thomas, E.; Francis, A. T.; Hill, A. H.; Fu, D. Biomed. Opt Express 2019, 10, 3860-3874. 